\title{
Phase Equilibrium of the Ternary System Consisting of Two Monodisperse Polystyrenes and Cyclohexane
}

\author{
Michio Tsuyumoto, Yoshiyuki EINAGA, and Hiroshi Fujita \\ Department of Macromolecular Science, Osaka University, \\ Toyonaka, Osaka 560, Japan
}

(Received November 18, 1983)

\begin{abstract}
Cloud point curves, binodals, and separation factors were determined for ternary solutions consisting of two "monodisperse" polystyrenes $\left(M_{1}=4.53 \times 10^{4}, M_{2}=4.98 \times 10^{5}\right)$ in cyclohexane. Very small fractions of a higher molecular weight component in the polymer mixture were found to have striking effects on the cloud point curve, critical point, and threshold point. The separation factor depended on polymer molecular weight, indicating that the Flory-Huggins $\chi$ parameter for the ternary system studied is a function of the concentrations of the individual polymer components, and not simply of their total concentration.

KEY WORDS Polystyrene / Phase Separation / Cloud Point Curve / Binodal / Critical Point / Threshold Point / Ternary Solution / Separation Factor / Flory-Huggins $\chi$ Parameter /
\end{abstract}

The complexity of equilibrium behavior of quasibinary polymer solutions may vary considerably, depending on the system and external conditions. When using the familiar FloryHuggins-type expression to express the free energy of mixing, this behavior should be described and predicted quantitatively in terms of the Flory-Huggins interaction parameter $\chi .^{1}$ This parameter involves all the thermodynamic deviations of actual quasibinary solutions from the classic Flory-Huggins solution taken as the reference state. Much effort $^{2-5}$ over the past three decades has been directed to finding empirical expressions for $\chi$ consistent with observed phase relationships. However, no completely adequate expression has been found for treating even strictly binary polymer solutions, the simplest of polymer + solvent systems. The difficulty arises primarily from the fact $^{6,7}$ that $\chi$ for such solutions depends on the polymer molecular weight as well as concentration and temperature. Thus $\chi$ for quasibinary polymer solutions should depend on the molecular weight distri- bution of the polymer solute. It is also the problem as to whether $\chi$ of such solutions depends simply on the total polymer concentration or more generally on the concentrations of the individual polymer components.

To solve these problems, a series of phase separation experiments have been carried out on strictly ternary systems consisting of two monodisperse polystyrene samples and cyclohexane, which are the simplest of quasibinary polymer solutions. Hashizume et al. ${ }^{8}$ investigated a case in which the molecular weights of the polymer components 1 and 2 were $4.53 \times 10^{4}$ and $1.03 \times 10^{5}$, respectively, to determine whether the separation (or partition) factor $\sigma$ depends on molecular weight. They found $\sigma$ to be approximately equal for the components 1 and 2 , in contrast with the results from typical previous work ${ }^{9}$ dealing with polydisperse polymer samples. Hashizume et ll. $^{8}$ may have chosen polymer samples whose molecular weight ratio was too small (about 2.3) to detect the molecular 
weight dependence of $\sigma$. Consequently, we carried out similar phase separation experiment using a pair of monodisperse polystyrene samples differing in molecular weight by a factor of about 10 . The major experimental findings and conclusions from this experiment are described in the following.

\section{EXPERIMENTAL}

\section{Polystyrene Samples}

Two Toyo Soda's standard polystyrenes F4 and F40 were used, whose polymolecularity indicies $M_{w} / M_{n}$ were 1.01 and 1.05 , respectively, according to the supplier. These samples were divided into several fractions by fractional precipitation or extraction with benzene + methanol mixtures to narrow their molecular weight distributions. The main fractions of F40 were further subjected to column elution fractionation. The central portions of the final fractions thus obtained from F4 and F40 were chosen as the test samples for the present experiment. These samples are referred to as $\mathrm{f} 4$ and $\mathrm{f} 40$ hereafter.

GPC measurements confirmed that the molecular weight distributions of the test samples were sufficiently narrow for our purposes. The weight-average molecular weight $M_{w}$, determined by light scattering with a Fica 50 light scattering photometer using benzene at $25^{\circ} \mathrm{C}$ as the solvent, was 45300 and 498000 for $\mathrm{f} 4$ and $\mathrm{f} 40$, respectively.

\section{Preparation of Cyclohexane Solutions}

Binary Solutions. Each test sample was dried at $50^{\circ} \mathrm{C}$ under reduced pressure for more than two days. A proper amount of the dry sample was weighed into a cylindrical cell of $c a .5 \mathrm{~cm}^{3}$ capacity containing a magnetic stirrer chip, and again thoroughly dried at $50^{\circ} \mathrm{C}$ for more than two days to remove traces of moisture. A desired amount of purified cyclohexane was then poured into the cell in a dry bag filled with nitrogen, and the cell was sealed with a ground glass stopper. After weighing, the cell was further sealed with parafilm.

Ternary Solutions. Dried samples $\mathrm{f} 4$ and $\mathrm{f} 40$ mixed at a desired weight ratio were freezedried from a benzene solution. Since traces of benzene were found to greatly affect phaseequilibrium behavior, the polymer mixture was again freeze-dried from a cyclohexane solution. Then, the same procedure as described above for binary solutions was used to make up a test solution.

The total polymer weight fraction $w$ in the test solution and weight fraction $\xi_{4}$ of the sample $\mathrm{f} 4$ in the polymer mixture were determined gravimetrically. Here $\xi_{4}$ is expressed by

$$
\xi_{4}=w_{4} /\left(w_{4}+w_{40}\right)
$$

with $w_{4}$ and $w_{40}$ the weight fractions of the samples $\mathrm{f} 4$ and $\mathrm{f} 40$, respectively. The total polymer volume fraction $\phi$ was calculated from $w$ using the relation

$$
\phi=\left[1+\left(v_{0} / v_{\mathrm{P}}\right)\left(w^{-1}-1\right)\right]^{-1}
$$

where $v_{0}$ and $v_{\mathrm{P}}$ are the specific volumes of the pure solvent and polymer, respectively. We used $1.2924 \mathrm{~cm}^{3} \mathrm{~g}^{-1}$ for $v_{0}$ and $0.9343 \mathrm{~cm}^{3} \mathrm{~g}^{-1}$ for $v_{\mathrm{P}}$ at any temperatures studied, though they refer to $25^{\circ} \mathrm{C}$. It should be noted that $\phi$ is simply a converted weight fraction and is the basic composition variable in the framework of the Flory-Huggins theory.

\section{Determination of Cloud Points}

The cylindrical cell containing a given test solution was mounted on a metal rack suspended in the inner bath of a double water thermostat. The solution was warmed to 35$40^{\circ} \mathrm{C}$ and then allowed to cool naturally by switching-off the temperature regulation system for the inner water bath, while the outer water bath was maintained at about 0.1 or $0.2^{\circ} \mathrm{C}$ lower than the cloud point (estimated by a preliminary experiment). During this cooling process, the solution was continuously stirred to maintain its temperature and concentration uniformly. In this way, the rate of cooling 


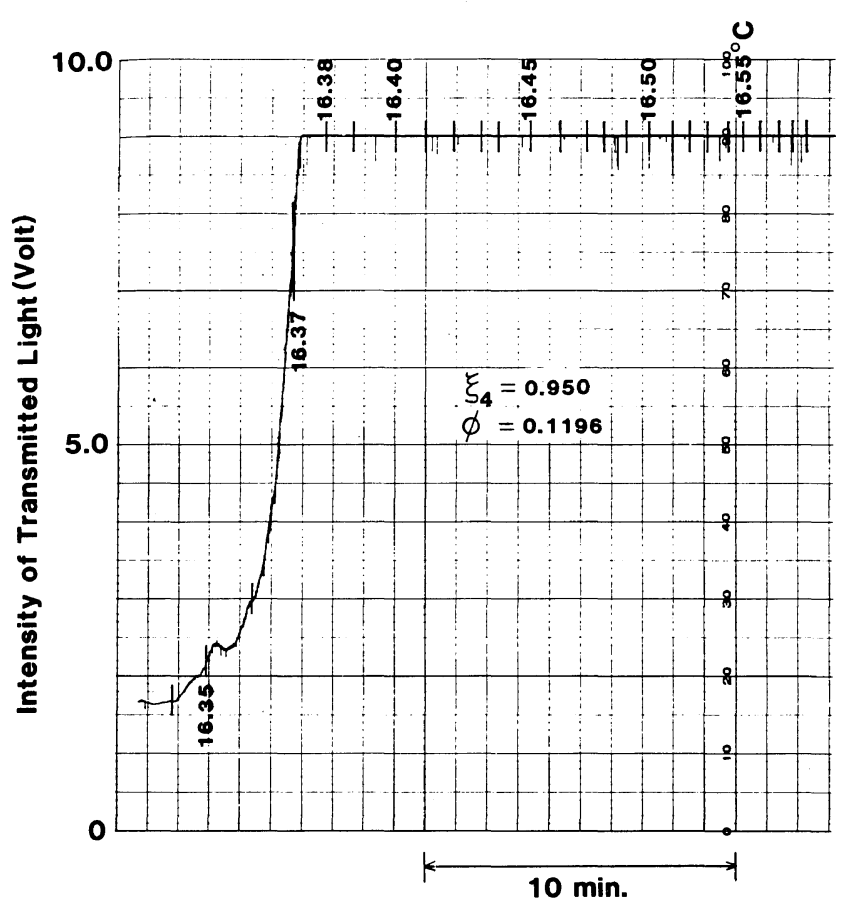

Figure 1. Cloud point determination for a polystyrene mixture $\mathrm{f} 4+\mathrm{f} 40\left(\xi_{4}=0.950\right)$ in cyclohexane at $\phi=$ 0.1196 .

could be slowed to about $0.01^{\circ} \mathrm{Cmin}^{-1}$ or less near the cloud point. A He-Ne laser beam was passed through the cooling solution, and the intensity of the transmitted light was monitored with a photodiode. Simultaneously, the temperature of the solution was measured at appropriate time intervals, using a Hewlett Packard Model 2802A or a Guildline Model 9535 digital thermometer. The intensity of the transmitted light decreased sharply when the temperature passed through the cloud point (see Figure 1). The cloud point was determined from the break point of the intensity $v s$. time curve, with an uncertainty of about $\pm 0.01^{\circ} \mathrm{C}$. This point was independent of the cooling rate slower than $0.01^{\circ} \mathrm{C} / \mathrm{min}$. Sometimes, cloud points were determined by visual observation of the appearance of speckles in the light path in the solution, and differed no more than $0.02^{\circ} \mathrm{C}$ from those determined by the more refined method using a laser beam.

\section{Determination of Conjugate Phases}

Phase separation was allowed to take place for a variety of initially uniform solutions at four temperatures from 14 to $25^{\circ} \mathrm{C}$. A solution of known $\phi$ was slowly cooled to each of these temperatures under continuous stirring and left standing at that temperature. In most cases, the solution separated into two liquid phases in equilibrium after one or two days. Attainment of equilibrium was reached when the height of the phase boundary ceased to change with time. The volume ratio of the upper to lower phase was determined from the height of the stationary phase boundary.

The GPC method described by Hashizume et al. ${ }^{8}$ was used to determine the total polymer concentration and polymer composition in each separated phase. Figure 2 illustrates these results for an original solution of $\xi_{4}=0.500$ separated into two phases of $\xi_{4}=0.883$ (upper phase) and $\xi_{4}=0.375$ (lower phase). The GPC peaks for samples $\mathrm{f} 4$ and $\mathrm{f} 40$ were completely 


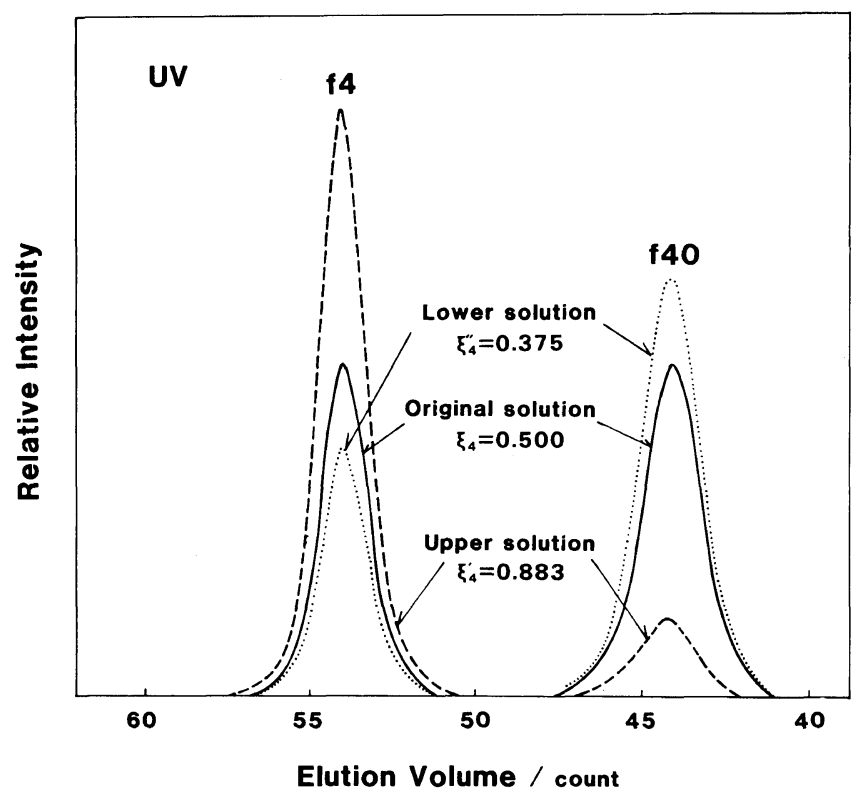

Figure 2. GPC curves for binary polystyrene mixtures in the two phases separated at $22^{\circ} \mathrm{C}$ from a cyclohexane solution with $\xi_{4}=0.500$ and $\phi=0.1006$.

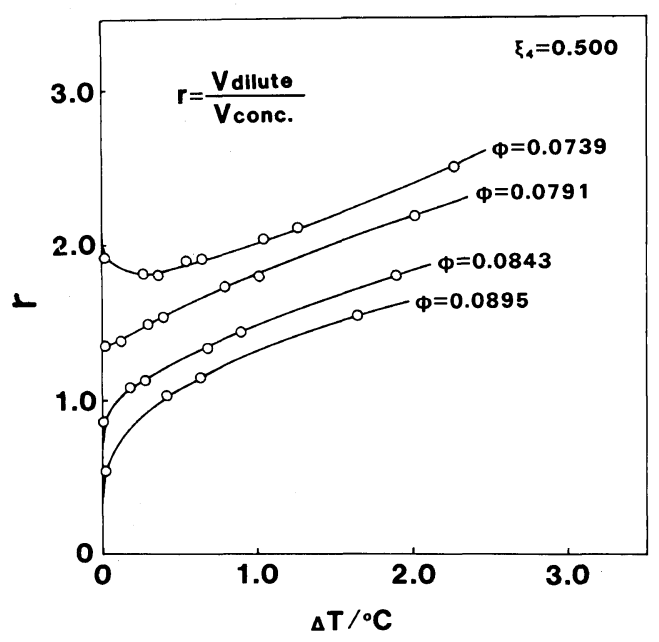

Figure 3a. Critical point determination for a polystyrene mixture $\mathrm{f} 4+\mathrm{f} 40$ with $\xi_{4}=0.500$ in cyclohexane.

separated and the position and half-width of each of these peaks remained unchanged before and after the phase separation. The latter finding confirms that both $\mathrm{f} 4$ and $\mathrm{f} 40$ may be treated as monodispersed. The polymer concentrations in the two phases were checked for

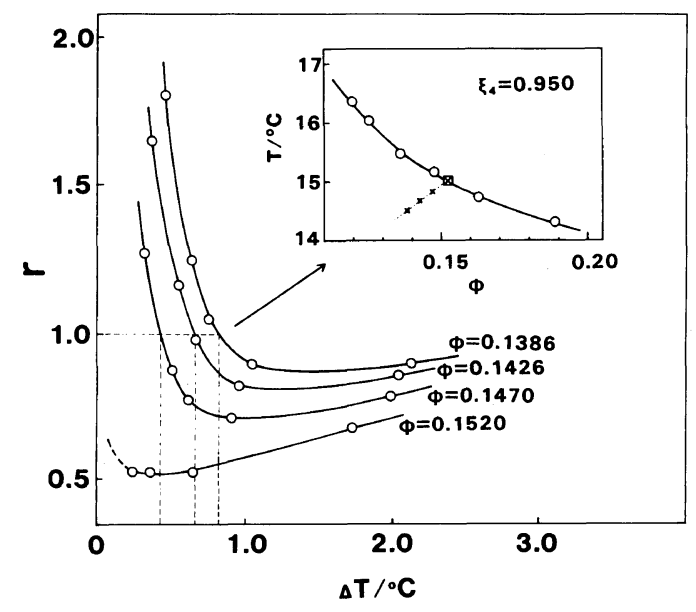

Figure 3b. Critical point determination for a polystyrene mixture $\mathrm{f} 4+\mathrm{f} 40$ with $\xi_{4}=0.950$ in cyclohexane (see the text for details).

adherence to the lever rule, using the phasevolume ratio data obtained.

\section{Determination of Critical Points}

For the temperature at which the binodal was experimentally determined, the critical 
concentration $\phi_{\mathrm{c}}$ was determined from the point on the composition triangle at which the locus of the midpoints of a series of tie lines intersected the binodal. For other temperatures, the familiar phase-volume ratio meth$\mathrm{od}^{10}$ was used in the following way to determine $\phi_{\mathrm{c}}$.

For a chosen $\xi_{4}$ a guess was made as to the critical temperature $T_{\mathrm{c}}$ and $\phi_{\mathrm{c}}$. Several solutions having the chosen $\xi_{4}$ but different $\phi$ near the guessed critical concentration were prepared. Each solution was allowed to phase separate at various temperatures near the guessed critical temperature, and the phasevolume ratios $r$ at the respective phase separation temperatures were determined. The plots of $r v s . \Delta T$ so obtained for $\xi_{4}=0.500$ and four different $\phi$ are shown in Figure 3a, and the corresponding plots for $\xi_{4}=0.950$ are shown in Figure $3 \mathrm{~b}$. Here, $\Delta T$ denotes the difference between the phase separation temperature and the cloud point, both referring to the chosen $\xi_{4}$ and $\phi$.

We know by theory that the $\phi$ value for a particular $r$ vs. $\Delta T$ curve converging to $r=1$ at $\Delta T=0$ gives $\phi_{\mathrm{c}}$ for the chosen $\xi_{4}$ and the cloud point associated with this curve is equal to $T_{\mathrm{c}}$ for the same $\xi_{4}$. Thus, we find from Figure $3 \mathrm{a}$ that $\phi_{\mathrm{c}}$ for $\xi_{4}=0.500$ lies between 0.0791 and 0.0843 . In fact, we determined $\phi_{\mathrm{c}}$ for this $\xi_{4}$ to be 0.082 . The data shown in Figure $3 b$ does not readily allow estimation of $\phi_{\mathrm{c}}$ for $\xi_{4}=$ 0.950 . Hence, the method of Kuwahara et al. ${ }^{11}$ was used. A horizontal line was drawn at $r=1$ and the values of $\Delta T$ at its intersections with the $r$ vs. $\Delta T$ curves for different $\phi$ were determined. The phase separation temperature was then calculated from each of these $\Delta T$ values and plotted against the corresponding $\phi$. The result is shown by a cross in the insert of Figure $3 \mathrm{~b}$. The square defines the point at which the dotted line connecting the crosses intersects the cloud point curve for $\xi_{4}=0.950$, giving the desired values of $T_{\mathrm{c}}$ and $\phi_{\mathrm{c}}$ for this value of $\xi_{4}$.

\section{RESULTS AND DISCUSSION}

\section{Cloud Point Curves}

Figure 4 shows the cloud point data for the ternary and binary solutions studied. The data points for the binary solutions of the sample $f 4$ include Hashizume et al.'s data ${ }^{8}$ on an essentially identical polystyrene sample in cyclohexane. The filled circles were derived from binodals (see below) by the method described previously, ${ }^{8}$ while the unfilled circles were obtained by direct cloud point measurement. The squares indicate the critical points. It can be seen that the critical temperature $T_{\mathrm{c}}$ falls monotonically but the critical concentration $\phi_{\mathrm{c}}$ passes through a maximum as $\xi_{4}$ increases from zero to unity. This behavior of $\phi_{\mathrm{c}}$ was also observed by Hashizume et al., ${ }^{8}$ though less clearly, probably because the molecular weight ratio of our polymer components was about 5 times that of theirs.

The most significant aspect of Figure 4 is the systematic change in the curvature of the branch of each cloud point curve to the right of the threshold point (the top of the cloud point curve) with an increase in $\xi_{4}$. This branch remains convex upward for $\xi_{4}$ smaller than about 0.40 , but shows a convexdownward part, i.e., a dent, for larger $\xi_{4}$. As $\xi_{4}$ approaches unity, the dent becomes deeper, eventually making the branch appear sharply bent at a point near the critical point. Surprisingly, even at $\xi_{4}=0.990$, the dent is still quite distinct and the branch to the left of the break point appears far above the cloud point curve for $\xi_{4}=1$ (pure F4). It should be noted that, at this $\xi_{4}$ value, the branch to the right of the break is almost indistinguishable from the curve for $\xi_{4}=1$. Thus, we see that the phaseseparation behavior of our ternary solution in the region of $\phi$ below $\phi_{\mathrm{c}}$ is extremely sensitive to $\xi_{4}$ when it is close to unity, i.e., when the polymer mixture contains a small amount of the higher molecular weight component. The cause of this high sensitivity is not yet clear, but its elucidation should substantially provide 


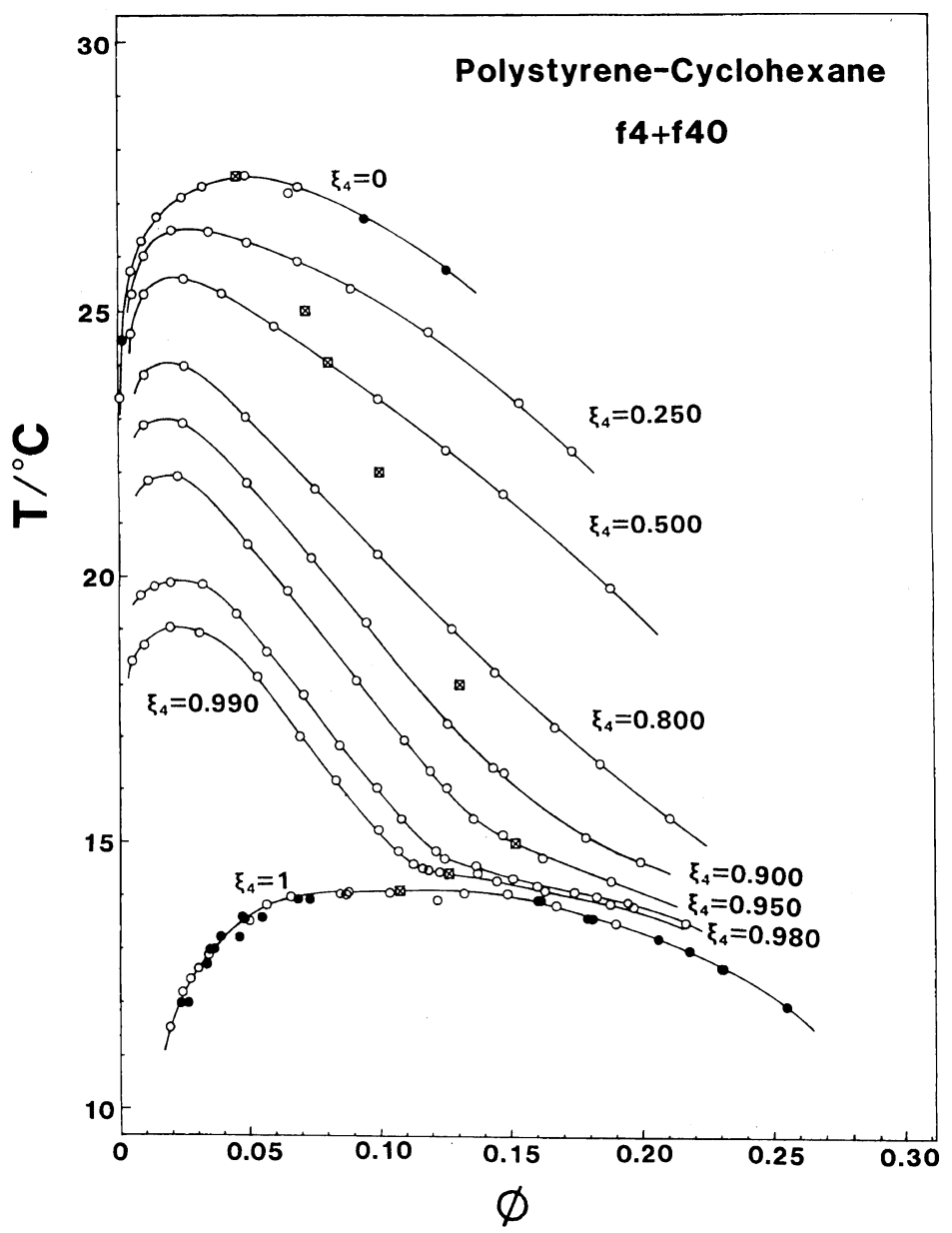

Figure 4. Cloud point curves for cyclohexane solutions of polystyrene mixtures $f 4+f 40$ with indicated $\xi_{4}$. Unfilled circles, by direct determination; filled circles, from binodals; squares, critical points.

a better understanding of polydispersity effects on the phase relationships of quasibinary polymer solutions.

Cloud point curves having such a dent have already been reported by several authors, ${ }^{2,8,12-14}$ since they were observed by Shultz and Flory ${ }^{12}$ for polystyrene mixtures in cyclohexane. However, the dents in our cloud point curves are far more pronounced than those reported so far.

Our molecular weight ratio of 10 was comparable to Tompa's critical value ${ }^{15}$ for the onset of three-liquid-phase separation in the type of ternary solution studied here. How- ever, we found no evidence for such multiphase separation in the ranges of $\phi$ and $T$ investigated.

\section{Binodals}

Table I summarizes the composition data for conjugate phases separated from a variety of original solutions at four temperatures. Figures 5 and 6 illustrate these data at 22 and $14^{\circ} \mathrm{C}$ plotted on the composition triangle, where $\phi_{4}$ and $\phi_{10}$ are the volume fractions of the samples $\mathrm{f} 4$ and $\mathrm{f} 40$, respectively, calculated by substituting $w_{4}$ and $w_{40}$ for $w$ in eq 2 . The unfilled circles indicate the cloud points in- 
Table I. Phase-composition data for the system $\mathrm{f} 4+\mathrm{f} 40$ in cyclohexane

\begin{tabular}{|c|c|c|c|c|c|c|c|}
\hline$\xi_{4}{ }^{\mathrm{a}}$ & $\phi^{\mathrm{a}}$ & $\phi_{4}^{\prime}$ & $\phi_{40}^{\prime}$ & $\phi_{4}{ }^{\prime \prime}$ & $\phi_{40}^{\prime \prime}$ & $\sigma_{4} \times 10^{3}$ & $\sigma_{40} \times 10^{3}$ \\
\hline \multicolumn{8}{|l|}{$14^{\circ} \mathrm{C}$} \\
\hline 0.250 & 0.02043 & 0.004127 & - & 0.02500 & 0.3084 & 4.63 & - \\
\hline 0.250 & 0.06985 & 0.009181 & - & 0.05735 & 0.2808 & 4.71 & - \\
\hline 0.250 & 0.1543 & 0.01035 & - & 0.07791 & 0.2529 & 5.19 & - \\
\hline 0.500 & 0.1261 & 0.02341 & - & 0.1312 & 0.1714 & 4.43 & - \\
\hline 0.500 & 0.1886 & 0.02455 & - & 0.1458 & 0.1610 & 4.58 & - \\
\hline 0.800 & 0.04945 & 0.03103 & - & 0.1639 & 0.1217 & 4.28 & - \\
\hline 0.800 & 0.1281 & 0.04701 & - & 0.1909 & 0.06006 & 3.60 & - \\
\hline 0.900 & 0.09531 & 0.05251 & - & 0.1858 & 0.03788 & 3.25 & - \\
\hline 0.900 & 0.1438 & 0.06570 & - & 0.1865 & 0.02456 & 2.68 & - \\
\hline 0.900 & 0.1793 & 0.06133 & - & 0.1947 & 0.02294 & 2.97 & - \\
\hline 0.950 & 0.06547 & 0.05151 & - & 0.1894 & 0.04067 & 3.35 & - \\
\hline 0.950 & 0.1196 & 0.06449 & - & 0.1956 & 0.01773 & 2.85 & - \\
\hline 0.990 & 0.1379 & 0.0720 & - & 0.1863 & 0.002148 & 2.44 & - \\
\hline 0.990 & 0.1451 & 0.0738 & - & 0.1778 & 0.001850 & 2.26 & - \\
\hline \multicolumn{8}{|l|}{$18^{\circ} \mathrm{C}$} \\
\hline 0.250 & 0.04994 & 0.01019 & - & 0.02420 & 0.2464 & 2.22 & - \\
\hline 0.500 & 0.04023 & 0.01745 & - & 0.05030 & 0.2090 & 2.72 & - \\
\hline 0.500 & 0.1482 & 0.04387 & 0.000425 & 0.09793 & 0.1294 & 2.06 & 1.34 \\
\hline 0.800 & 0.07603 & 0.05380 & 0.000445 & 0.1199 & 0.09244 & 2.06 & 1.25 \\
\hline 0.800 & 0.09980 & 0.06945 & 0.001259 & 0.1356 & 0.08025 & 1.72 & 0.971 \\
\hline 0.800 & 0.1446 & 0.1095 & 0.01208 & 0.1261 & 0.03607 & 0.363 & 0.256 \\
\hline \multicolumn{8}{|l|}{$22^{\circ} \mathrm{C}$} \\
\hline 0.250 & 0.03497 & 0.007735 & 0.000516 & 0.01695 & 0.1834 & 2.02 & 1.37 \\
\hline 0.250 & 0.09011 & 0.01612 & 0.001696 & 0.03166 & 0.1633 & 1.73 & 1.07 \\
\hline 0.500 & 0.06042 & 0.02658 & 0.001894 & 0.04486 & 0.1407 & 1.34 & 1.01 \\
\hline 0.500 & 0.08129 & 0.03400 & 0.003412 & 0.05575 & 0.1206 & 1.27 & 0.833 \\
\hline 0.500 & 0.1006 & 0.04097 & 0.005413 & 0.06217 & 0.1038 & 1.07 & 0.690 \\
\hline 0.500 & 0.1222 & 0.05034 & 0.008989 & 0.06663 & 0.08167 & 0.720 & 0.516 \\
\hline 0.670 & 0.09009 & 0.05724 & 0.02170 & 0.06747 & 0.06585 & 0.423 & 0.259 \\
\hline \multicolumn{8}{|l|}{$25^{\circ} \mathrm{C}$} \\
\hline 0.237 & 0.04458 & 0.09918 & 0.006524 & 0.01359 & 0.1104 & 0.809 & 0.661 \\
\hline 0.237 & 0.09677 & 0.02003 & 0.01485 & 0.02529 & 0.08739 & 0.599 & 0.414 \\
\hline 0.237 & 0.07146 & 0.01440 & 0.009165 & 0.02003 & 0.1008 & 0.848 & 0.560 \\
\hline 0.485 & 0.04944 & 0.02300 & 0.02026 & 0.03154 & 0.06676 & 0.811 & 0.279 \\
\hline
\end{tabular}

a Solution composition before phase separation.

terpolated from the smooth curves in Figure 4, while the filled circles represent the directly measured compositions of conjugate phases. Both filled and unfilled points can be fitted well by a single curve (Figure 5), justifying our basic assumption that samples $\mathrm{f} 4$ and $\mathrm{f} 40$ may be regarded as monodispersed.

In Figure 5, the four crosses on the dot-dash line indicate the original solutions having the same $\xi_{4}$ but different $\phi$, each eventually separating into conjugate phases connected by a tie line. The dash line joins the midpoints of a series of tie lines and defines the critical point at its intersection with the binodal.

The tie lines at $22^{\circ} \mathrm{C}$ for different $\xi_{4}$ and $\phi$ (each cross indicates these two quantities) remain almost parallel to the $\phi_{40}$ axis (Figure 5), whereas those at $14^{\circ} \mathrm{C}$ change their direction from the $\phi_{40}$ axis to the $\phi_{4}$ axis as $\xi_{4}$ increases from zero to unity (Figure 6). The binodal at $14^{\circ} \mathrm{C}$ intersects the $\phi_{4}$ axis at a pair of points, though only one for the higher $\phi_{4}$ can be 


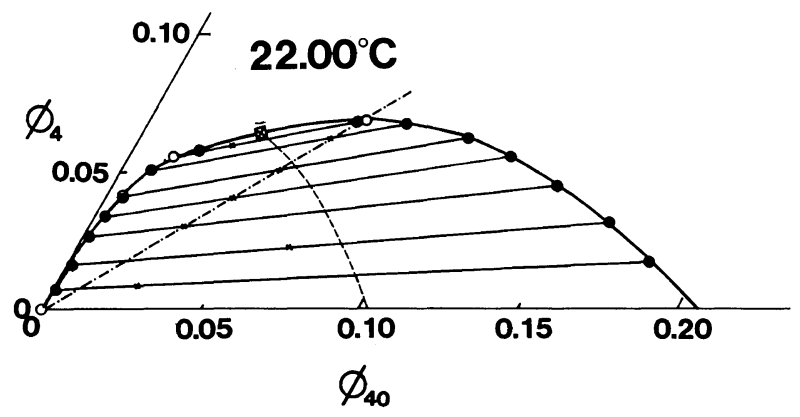

Figure 5. Binodal for the ternary system $\mathrm{f} 4+\mathrm{f} 40+$ cyclohexane at $22^{\circ} \mathrm{C}$. Unfilled circles, from cloud point curves; filled circles, from composition analysis; crosses, solutions before phase separation; square, critical point. Solid curve, binodal; thin straight lines, tie lines; dashed curve, locus of the midpoints of tie lines; dot-dash line, composition line for $\xi_{4}=0.500$.

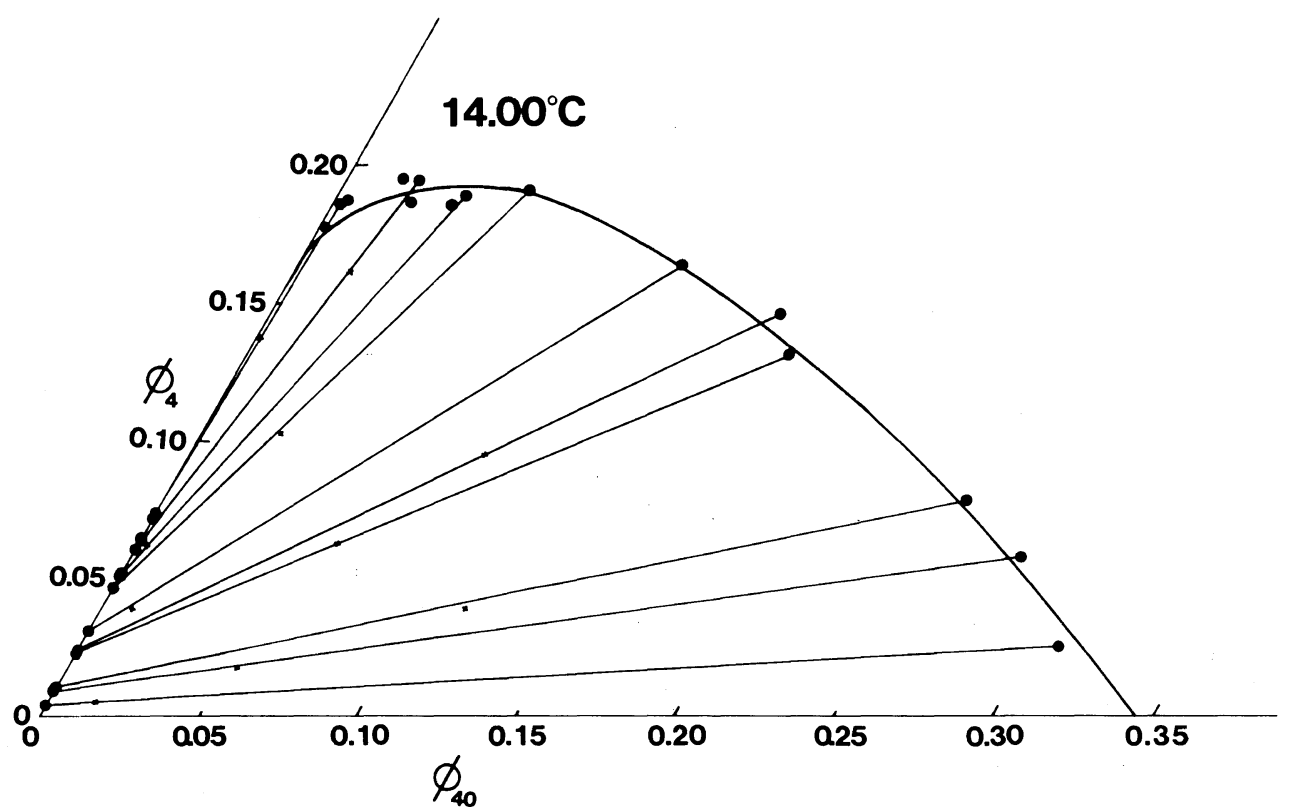

Figure 6. Binodal for the ternary system $\mathrm{f} 4+\mathrm{f} 40+$ cyclohexane at $14^{\circ} \mathrm{C}$. The symbols and lines have the same meaning as in Figure 5.

identified clearly in this figure. Thus, the binary solution of $\mathrm{f} 4$ is phase separated at $14^{\circ} \mathrm{C}$.

The four binodals obtained, along with the critical points, are shown in Figure 7 to illustrate how the binodal changes with temperature. In the open region to the right of the dash line connecting the critical points, the binodal expands almost uniformly with lowering temperature, giving a larger demixing area at a lower temperature. From this feature, it follows that, in the region of $\phi$ above $\phi_{c}$, the cloud point curves for different $\xi_{4}$ (except those at $\xi_{4}$ close to unity) are nearly parallel to that for $\xi_{4}=0$, as evident from Figure 4 .

In the corner region to the left of the dash line in Figure 7, the binodal extends toward the $\phi_{4}$ axis. In particular, those at 18 and $22^{\circ} \mathrm{C}$ appear to touch the $\phi_{4}$ axis tangentially, but, in fact, there is some finite clearance from the $\phi_{4}$ axis. It thus follows that even a composition line almost coinciding with the $\xi_{4}$ axis, i.e., one for $\xi_{4} \sim 1$, intersects these binodals. This ex- 


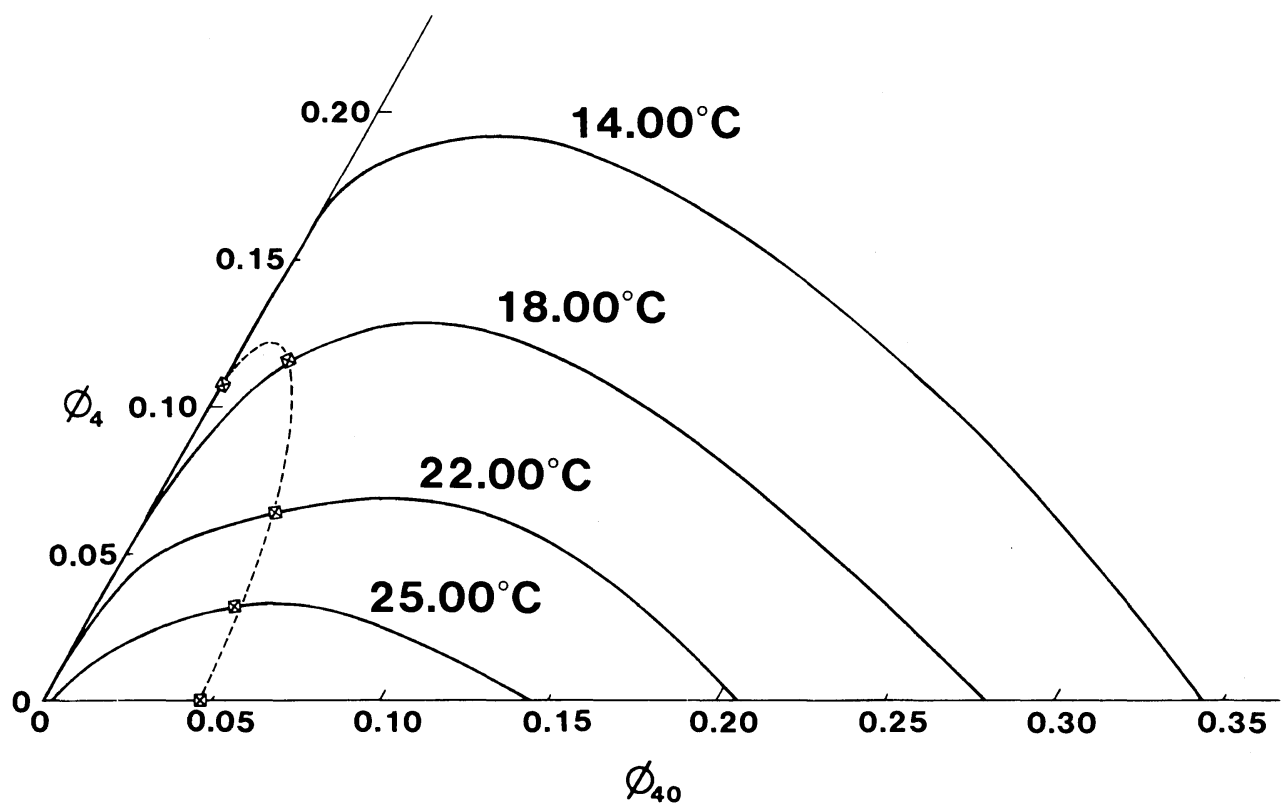

Figure 7. Solid lines, binodals for the ternary system $f 4+f 40+$ cyclohexane at the indicated temperatures. Dashed line, locus of critical points.

plains why, in the region of $\phi$ below $\phi_{c}$, the cloud point curves for $\xi_{4}$ close to unity go far above $T_{\mathrm{c}}$, as seen in Figure 4 . The binodals in Figure 7 are similar in overall shape to those obtained by Hashizume et al., ${ }^{8}$ but differ from the latter in that they extend to the more dilute region.

\section{Critical Points and Threshold Points}

Our data for critical points $\left(T_{\mathrm{c}}, \phi_{\mathrm{c}}\right)$ and threshold points $\left(T_{\mathrm{th}}, \phi_{\mathrm{th}}\right)$ are summarized in Table II, and, along with those of Hashizume et al., plotted against $\xi_{4}$ in Figures 8 and 9 . These figures show to what extent the $\xi_{4}$ dependence of critical and threshold behavior for the ternary system treated here varies as the molecular weight ratio $M_{2} / M_{1}$ of the polymer components increases from 2.3 (Hashizume et al.'s case ${ }^{8}$ ) to 10 (our case).

The following features should be noted.

(1) The $T_{\mathrm{c}}$ curve for Hashizume et al.'s case is convex upward over the entire range of $\xi_{4}$, while that for our case has an inflection at a $\xi_{4}$ value near 0.90 . In either case, the changes in
Table II. Critical and threshold points for the ternary system consisting of two "monodispersed" polystyrenes and cyclohexane

\begin{tabular}{|c|c|c|c|c|c|}
\hline$\xi_{4}$ & $\frac{T_{\mathrm{c}}}{{ }^{\circ} \mathrm{C}}$ & $\phi_{\mathrm{c}}$ & $\xi_{4}$ & $\frac{T_{\mathrm{th}}}{{ }^{\circ} \mathrm{C}}$ & $\phi_{\mathrm{th}}$ \\
\hline \multicolumn{6}{|c|}{$\mathrm{f} 4+\mathrm{f} 40$ (Present data) } \\
\hline 0 & 27.55 & 0.047 & 0 & 27.55 & 0.047 \\
\hline 0.436 & 25.00 & 0.073 & 0.25 & 26.5 & 0.026 \\
\hline 0.500 & 24.08 & 0.082 & 0.50 & 25.6 & 0.022 \\
\hline 0.64 & 22.00 & 0.100 & 0.80 & 24.1 & 0.020 \\
\hline 0.86 & 18.00 & 0.131 & 0.90 & 23.0 & 0.019 \\
\hline 0.950 & 15.01 & 0.152 & 0.95 & 21.9 & 0.020 \\
\hline 0.990 & 14.43 & 0.126 & 0.98 & 19.9 & 0.023 \\
\hline \multirow[t]{2}{*}{1} & 14.14 & 0.108 & 0.99 & 19.0 & 0.025 \\
\hline & & & 1 & 14.14 & 0.108 \\
\hline \multicolumn{6}{|c|}{$\mathrm{f} 4+\mathrm{f} 10\left(\right.$ Hashizume et al..$\left.^{8}\right)$} \\
\hline 0 & 20.64 & 0.086 & 0 & 20.64 & 0.086 \\
\hline 0.350 & 19.00 & 0.096 & 0.150 & 20.12 & 0.067 \\
\hline 0.532 & 18.00 & 0.101 & 0.300 & 19.34 & 0.057 \\
\hline 0.685 & 17.00 & 0.105 & 0.501 & 18.36 & 0.050 \\
\hline 0.78 & 16.00 & 0.110 & 0.651 & 17.50 & 0.052 \\
\hline 0.867 & 15.71 & 0.114 & 0.850 & 16.00 & 0.055 \\
\hline 0.885 & 15.39 & 0.113 & 1 & 14.14 & 0.112 \\
\hline 1 & 14.14 & 0.112 & & & \\
\hline
\end{tabular}




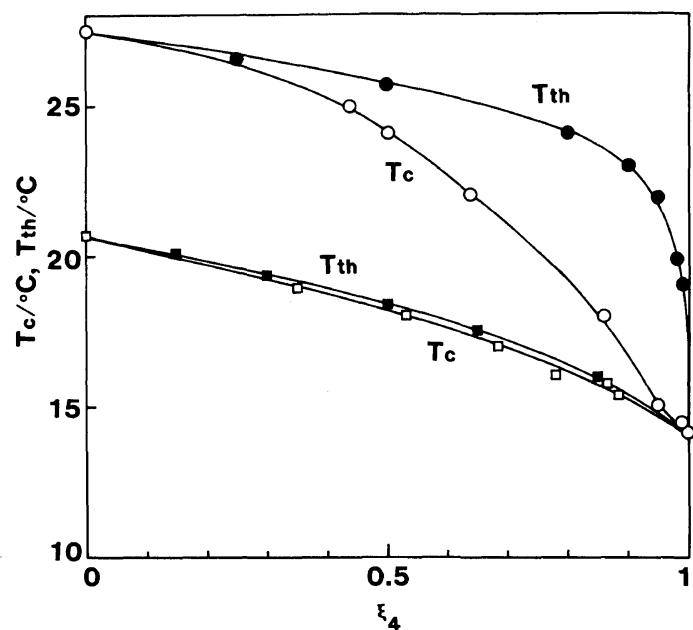

Figure 8. Critical temperature $T_{\mathrm{c}}$ and threshold temperature $T_{\text {th }}$ as functions of $\xi_{4}$ for binary polystyrene mixtures in cyclohexane. Circles, for mixtures $\mathrm{f} 4+\mathrm{f} 40$ (present data); squares, for mixtures $\mathrm{f} 4+\mathrm{f} 10$ (Hashizume et $\left.a .^{8}\right)$. Unfilled symbols, $T_{\mathrm{c}}$; filled symbols, $T_{\mathrm{th}}$.

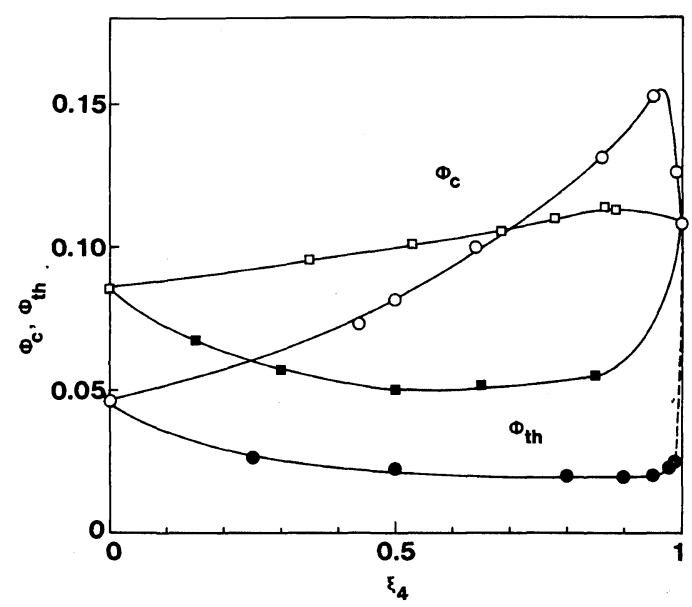

Figure 9. Critical concentrations $\phi_{\mathrm{c}}$ and threshold concentrations $\phi_{\mathrm{th}}$ for binary polystyrene mixtures in cyclohexane. Circles, for mixtures $\mathrm{f} 4+\mathrm{f} 40$ (present data); squares, for mixtures $\mathrm{f} 4+\mathrm{f} 10$ (Hashizume et al. ${ }^{8}$ ). Unfilled symbols, $\phi_{\mathrm{c}}$; filled symbols, $\phi_{\mathrm{th}}$.

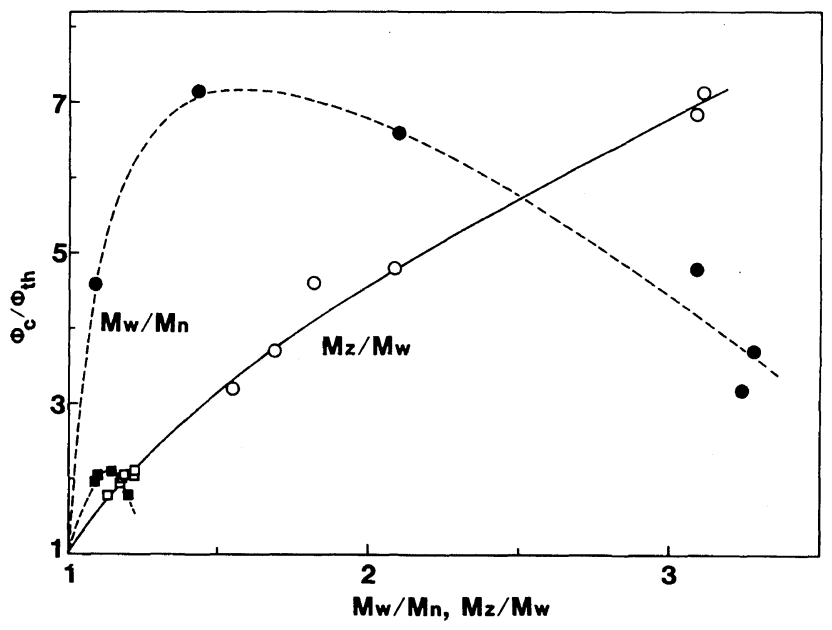

Figure 10. Ratio $\phi_{\mathrm{c}} / \phi_{\mathrm{th}}$ for binary polystyrene mixtures in cyclohexane plotted against $M_{w} / M_{n}$ (filled symbols) and $M_{z} / M_{w}$ (unfilled symbols). Circles, for mixtures $\mathrm{f} 4+\mathrm{f} 40$ (present data); squares, for mixtures $\mathrm{f} 4+\mathrm{f} 10$ (Hashizume et al. ${ }^{8}$ ).

$T_{\mathrm{c}}$ with $\xi_{4}$ are monotonic and gradual.

(2) In Hashizume et al.'s case, $T_{\text {th }}$ almost agrees with $T_{\mathrm{c}}$ throughout the entire range of $\xi_{4}$, while, in our case, $T_{\text {th }}$ appears far above $T_{\mathrm{c}}$ over a substantial range of $\xi_{4}$. The fall of $T_{\text {th }}$ for the latter in the region near $\xi_{4}=1$ is too sharp to be determined accurately.

(3) In either case, $\phi_{c}$ shows a maximum at a $\xi_{4}$ near unity. The maximum is very pronounced in our case.

(4) In either case, $\phi_{\text {th }}$ passes through a broad minimum. It is interesting that, with increasing $\xi_{4}$, the threshold point first moves toward a lower concentration. Also of interest is the finding that $\phi_{\mathrm{th}}$ for our case remains far below the value at $\xi_{4}=1$ until $\xi_{4}$ comes very close to 
unity. Thus, in the vicinity of $\xi_{4}=1$, our $\phi_{\text {th }} v s$. $\xi_{4}$ curve has an almost infinite slope (see the dash line in Figure 9).

From these data, we find that when $M_{2} / M_{1}$ is as large as 10 , a very small fraction of the higher molecular weight component has a striking effect on $\phi_{\mathrm{c}}, T_{\mathrm{th}}$, and $\phi_{\mathrm{th}}$ for the ternary system under consideration. In this connection, we note that our experimental curves for $T_{\mathrm{c}}$ and $\phi_{\mathrm{c}}$ are comparable to those derived theoretically by $\operatorname{Vink}^{16}$ for $M_{2} / M_{1}=10$ under variously assigned $M_{1}$ and $M_{2}$ values. Unfortunately he did not treat the threshold point.

Solc $^{17}$ has investigated theoretically the dependence of $\phi_{\mathrm{c}} / \phi_{\mathrm{th}}$ for quasibinary polymer solutions on the polydispersity indicies $M_{w} / M_{n}, M_{z} / M_{w}$, and so forth, confining himself to the special case in which Flory-Huggins interaction parameter $\chi$ is independent of concentration. In Figure 10, the values of $\phi_{\mathrm{c}} / \phi_{\mathrm{th}}$ computed from Hashizume et al.'s data and ours are plotted against both $M_{w} / M_{n}$ and $M_{z} / M_{w}$. Clearly, the data points have no correlation with $M_{w} / M_{n}$, in agreement with Solc's conclusion that critical and threshold behavior has nothing to do with $M_{n}$. On the other hand, it appears that $\phi_{\mathrm{c}} / \phi_{\mathrm{th}}$ depends approximately on $M_{z} / M_{w}$ only.

\section{Separation Factors}

Table I also shows the values of the separation factors $\sigma_{4}$ and $\sigma_{40}$ for the samples $\mathrm{f} 4$ and f40; these were computed by substituting the tabulated values of $\phi_{i}{ }^{\prime}$ and $\phi_{i}{ }^{\prime \prime}(i=4,40)$ into the defining equation

$$
\sigma_{i}=\left(1 / P_{i}\right) \ln \left(\phi_{i}{ }^{\prime \prime} / \phi_{i}{ }^{\prime}\right)
$$

where $P_{i}$ is the relative chain length of the sample $i$. It can be seen that, except for a few cases, the difference $\sigma_{4}-\sigma_{40}$ amounts to values as large as $35 \%$ of $\sigma_{4}$, in contrast to the case $(\mathrm{f} 4+\mathrm{f} 10$ in cyclohexane) studied by Hashizume et al., ${ }^{8}$ in which the difference $\sigma_{4}-\sigma_{10}$ was less than $10 \%$ of $\sigma_{4}$ or essentially negligible within experimental accuracy. Thus, we may conclude that the separation factor is molecular-weight dependent, as has been argued by previous authors ${ }^{9}$ and that, as noted in the Introduction, Hashizume et al. ${ }^{8}$ chose the polymer samples whose molecular weights were too close to observe this dependence clearly.

The molecular weight dependence of the separation factor introduces greater complexity into the theoretical treatment of quasibinary polymer solutions, since it implies that the parameter $\chi$ depends not on the total concentration of the solute but on the concentrations of the individual polymer components. ${ }^{18}$

Acknowledgment. The authors wish to thank Dr. M. Fukuda of Toyo Soda Co., for kindly providing the polystyrene samples used in this study.

\section{REFERENCES}

1. M. Kurata, "Thermodynamics of Polymer Solutions," translated from the Japanese by H. Fujita, Harwood Academic, N. Y., 1982.

2. R. Koningsveld, Thesis, Leiden University, 1967.

3. R. Koningsveld and L. A. Kleintjens, Macromolecules, 4, 637 (1971).

4. R. Koningsveld, W. H. Stockmayer, J. W. Kennedy, and L. A. Kleintjens, Macromolecules, 7, 73 (1974).

5. H. Fujita and A. Teramoto, Proceedings of MMI Symposium on Polymer Compatibility and Imcompatibility, Harwood Academic, N. Y., 1982, p 125.

6. TH. G. Scholte, Eur. Polym. J., 6, 1063 (1970).

7. Y. Einaga, S. Ohashi, Z. Tong, and H. Fujita, Macromolecules, to be published.

8. J. Hashizume, A. Teramoto, and H. Fujita, J. Polym. Sci., Polym. Phys. Ed., 19, 1405 (1981).

9. L. A. Kleintjens, R. Koningsveld, and W. H. Stockmayer, Br. Polym. J., 8, 144 (1976).

10. R. Koningsveld, L. A. Kleintjens, and A. R. Shultz, J. Polym. Sci., A-2, 8, 1261 (1970).

11. N. Kuwahara, M. Nakata, and M. Kaneko, Polymer, 14, 415 (1973).

12. A. R. Shultz and P. J. Flory, J. Am. Chem. Soc., 74, 4760 (1952).

13. G. Rehage, D. Moller, and O. Ernst, Makromol. Chem., 88, 232 (1965).

14. G. Rehage and R. Koningsveld, J. Polym. Sci., Polym. Lett. Ed., 6, 421 (1968). 
M. Tsuyumoto, Y. Einaga, and H. Fujita

15. H. Tompa, Trans. Faraday Soc., 45, 1142 (1949).

16. H. Vink, Eur. Polym. J., 12, 77 (1976).

17. K. Solc, J. Polym. Sci., 12, 1865 (1974).
18. H. Fujita and A. Teramoto, J. Polym. Sci., Polym. Phys. Ed., 20, 893 (1982). 